\title{
ON THE FINITENESS OF THE SET OF HILBERT COEFFICIENTS
}

\author{
SHREEDEVI K. MASUTI AND KUMARI SALONI
}

\begin{abstract}
Let $(R, \mathfrak{m})$ be a Noetherian local ring of dimension $d$ and $K, Q$ be m-primary ideals in $R$. In this paper we study the finiteness properties of the sets $\Lambda_{i}^{K}(R):=\left\{g_{i}^{K}(Q)\right.$ : $Q$ is a parameter ideal of $R\}$, where $g_{i}^{K}(Q)$ denotes the Hilbert coefficients of $Q$ with respect to $K$, for $1 \leq i \leq d$. We prove that $\Lambda_{i}^{K}(R)$ is finite for all $1 \leq i \leq d$ if and only if $R$ is generalized Cohen-Macaulay. Moreover, we show that if $R$ is unmixed then finiteness of the set $\Lambda_{1}^{K}(R)$ suffices to conclude that $R$ is generalized Cohen-Macaulay. We obtain partial results for $R$ to be Buchsbaum in terms of $\left|\Lambda_{i}^{K}(R)\right|=1$. Our results are more general than in [GGH $\left.{ }^{+} 15\right]$ and [GO11]. We also obtain a criterion for the set $\Delta^{K}(R):=\left\{g_{1}^{K}(I): I\right.$ is an $\mathfrak{m}$-primary ideal of $\left.R\right\}$ to be finite, generalizing a result of [KT15].
\end{abstract}

\section{INTRODUCTION}

The main objective of this paper is to study the finiteness properties of various sets of the Hilbert coefficients relative to the properties of the ring. First we introduce the notations needed to define these sets.

Throughout this paper $(R, \mathfrak{m})$ denotes a Noetherian local ring of dimension $d$ with maximal ideal $\mathfrak{m}, M$ a finitely generated $R$-module of dimension $r$ and $K$ a fixed $\mathfrak{m}$-primary ideal.

For an m-primary ideal $Q$, the fiber cone of $Q$ with respect to $K$ is the standard graded algebra $F_{K}(Q)=\underset{n \geq 0}{\oplus} Q^{n} / K Q^{n}$. The Hilbert function of the fiber cone $F_{K}(Q)$ is given by $H(F, n):=$ $\ell_{R}\left(Q^{n} / K Q^{n}\right)$, where $\ell_{R}(M)$ denotes the length of an $R$-module $M$. It is well known that $H(F, n)$ agrees with a polynomial $P(F, n)$ of degree $d-1$, for $n \gg 0$, called the Hilbert polynomial of $F_{K}(Q)$. We can write $P(F, n)$ in the following way:

$$
P(F, n)=\sum_{i=0}^{d-1}(-1)^{i} f_{i}^{K}(Q)\left(\begin{array}{c}
n+d-i-1 \\
d-1-i
\end{array}\right)
$$

where the coefficients $f_{i}^{K}(Q)$ are integers known as the fiber coefficients of $Q$ with respect to $K$. The Hilbert-Samuel function of $Q$ for $M$ is the function $H(Q, n, M)=\ell_{R}\left(M / Q^{n} M\right)$. In [JV05a] authors introduced the Hilbert function of $Q$ with respect to $K$ defined as $H_{K}(Q, n)=$ $\ell_{R}\left(R / K Q^{n}\right)$. It is known that for $n \gg 0, H(Q, n, M)$ (resp. $\left.H_{K}(Q, n)\right)$ agrees with a polynomial $P(Q, n, M)$ (resp. $\left.P_{K}(Q, n)\right)$ of degree $r$ (resp. $d$ ). We can write these polynomials in the following manner:

$$
\begin{aligned}
P(Q, n, M) & =\sum_{i=0}^{r}(-1)^{i} e_{i}(Q, M)\left(\begin{array}{c}
n+r-i-1 \\
r-i
\end{array}\right) \\
P_{K}(Q, n) & =\sum_{i=0}^{d}(-1)^{i} g_{i}^{K}(Q)\left(\begin{array}{c}
n+d-i-1 \\
d-i
\end{array}\right)
\end{aligned}
$$

Date: October 21, 2018.

2010 Mathematics Subject Classification. Primary: 13H10, 13D40; Secondary: 13D45.

Key words and phrases. Hilbert coefficients, fiber coefficients, Buchsbaum module, generalized Cohen-Macaulay module.

The first author is supported by INdAM COFOUND Fellowships cofounded by Marie Curie actions, Italy. 
for unique integers $e_{i}(Q, M)$ (resp. $\left.g_{i}^{K}(Q)\right)$ known as the Hilbert coefficients of $Q$ for $M$ (resp. Hilbert coefficients of $Q$ with respect to $K)$. One of the motivations to study $g_{i}^{K}(Q)$ is that these coefficients are related to the fiber coefficients (see (2.3)) and hence are useful to study the properties of $f_{i}^{K}(Q)$. The properties of $g_{i}^{K}(Q)$ have been studied in [D'cr13], [GZT07], [JV05a], [JV05b], [Sal16], [ZGT08].

In this paper we consider the sets

$$
\begin{aligned}
\Lambda_{i}^{K}(R) & =\left\{g_{i}^{K}(Q) \mid Q \text { is a parameter ideal of } R\right\} \text { and } \\
\delta_{i}^{K}(R) & =\left\{g_{i}^{K}(Q) \mid Q \text { is a parameter ideal of } R \text { such that } Q \subseteq K\right\}
\end{aligned}
$$

for $1 \leq i \leq d$. Note that $\delta_{i}^{K}(R) \subseteq \Lambda_{i}^{K}(R)$. Following the notation of [GGH $\left.{ }^{+} 15\right]$, we set

$$
\Lambda_{i}(M)=\left\{e_{i}(Q, M) \mid Q \text { is a parameter ideal for } M\right\} \text { for all } 1 \leq i \leq r .
$$

For a set $S$ we use $|S|$ to denote the cardinality of the set $S$.

Let $r, d \geq 2$. In [GGH $\left.{ }^{+} 15\right]$ authors proved that the set $\Lambda_{1}(M)$ is finite (resp. singleton) if and only if $M$ is generalized Cohen-Macaulay (resp. Buchsbaum) provided $M$ is an unmixed module, see [GGH ${ }^{+} 15$, Theorems 4.5 and 5.4]. In Section 3, we investigate the set $\Lambda_{1}^{K}(R)$ for analogous properties. We prove that an unmixed local ring $R$ is generalized Cohen-Macaulay if and only $\Lambda_{1}^{K}(R)$ ( equivalently $\delta_{1}^{K}(R)$ ) is finite (Theorem 3.2). Next, we prove that if $R$ is unmixed and $\left|\Lambda_{1}^{K}(R)\right|=1$ then $R$ is Buchsbaum where as the converse holds true for $K=\mathfrak{m}$ (Theorem 3.5). We expect that $\Lambda_{1}^{K}(R)$ need not be singleton in a Buchsbaum local ring for an arbitrary m-primary ideal $K$ (see Discussion 3.7).

In Section 4, we study the finiteness of the sets $\Lambda_{i}^{K}(R)$ for all $1 \leq i \leq d$. We prove that $R$ (need not be unmixed) is generalized Cohen-Macaulay if and only if $\Lambda_{i}^{K}(R)$ (equivalently $\left.\delta_{i}^{K}(R)\right)$ are finite for all $1 \leq i \leq d-1$ (Theorem 4.9). In [GO11, Theorem 1.1] authors proved that $R$ is generalized Cohen-Macaulay if and only if $\Lambda_{i}(R)$ is finite for all $1 \leq i \leq d$. We improve their result and extend it to modules. More precisely, we show that $M$ (resp. $M / \mathrm{H}_{\mathfrak{m}}^{0}(M)$ ) is generalized Cohen-Macaulay (resp. Buchsbaum) if and only if $\left|\Lambda_{i}(M)\right|<\infty$ (resp. $\left|\Lambda_{i}(M)\right|=1$ ) for all $1 \leq i \leq r-\operatorname{depth} M$ (Theorems 4.7 and 4.10).

In section 5 , we consider the set

$$
\Delta^{K}(R)=\left\{g_{1}^{K}(I) \mid I \text { is an } \mathfrak{m} \text {-primary ideal of } R\right\} .
$$

In [KT15, Theorem 1.1], authors proved that the set $\left\{e_{1}(I, R) \mid I\right.$ is an $\mathfrak{m}$-primary ideal of $\left.R\right\}$ is finite if and only if $d=1$ and $R / \mathrm{H}_{\mathfrak{m}}^{0}(R)$ is analytically unramified. We prove that $\Delta^{K}(R)$ is finite if and only if $d=1$ and $R / \mathrm{H}_{\mathfrak{m}}^{0}(R)$ is analytically unramified (Theorem 5.7).

We gather preliminary results needed in section 2 .

Few words about proofs. Considering $K$ as an $R$-module, we get a relation between $g_{i}^{K}(Q)$ and $e_{i}(Q, K)$ which shows that $\left|\Lambda_{i}^{K}(R)\right|=\left|\Lambda_{i}(K)\right|$ (See (2.1)). This suggests that results on the finiteness properties of the set $\Lambda_{i}(M)$, for any finitely generated module $M$, are useful to study the similar properties of $\Lambda_{i}^{K}(R)$. This method is used in order to study the finiteness of the set $\Lambda_{i}^{K}(R)$ in this paper. This method depends on the module theoretic properties of $K$ and is used to study the finiteness of the set $\Lambda_{i}^{K}(R)$ in this paper.

We refer [Mat06] and [BH93] for undefined terms.

\section{PRELIMINARIES}

In this section we prove some preliminary results needed in the subsequent sections. We first note a relation between the Hilbert coefficients and the fiber coefficients. 
Remark 2.1. (1) Let $d \geq 1$. Since $\ell_{R}\left(R / K Q^{n}\right)=\ell_{R}(R / K)+\ell_{R}\left(K / Q^{n} K\right)$, for all $n \in \mathbb{Z}$, $P_{K}(Q, n)=\ell_{R}(R / K)+P(Q, n, K)$. Thus comparing the coefficients of both sides, we get

$$
g_{0}^{K}(Q)=e_{0}(Q, K) \text { and } g_{i}^{K}(Q)= \begin{cases}e_{i}(Q, K) & \text { if } i \neq d \\ e_{d}(Q, K)+(-1)^{d} \ell_{R}(R / K) & \text { if } i=d .\end{cases}
$$

(2) Since $\ell_{R}\left(R / K Q^{n}\right)=\ell_{R}\left(R / Q^{n}\right)+\ell_{R}\left(Q^{n} / K Q^{n}\right)$ for all integers $n$, we have $P_{K}(Q, n)=$ $P(Q, n, R)+P(F, n)$ for all integers $n$. Thus comparing the coefficients of both sides, we get

$$
\begin{aligned}
& g_{0}^{K}(Q)=e_{0}(Q, R) \text { and } \\
& f_{i}^{K}(Q)=e_{i+1}(Q, R)-g_{i+1}^{K}(Q)+e_{i}(Q, R)-g_{i}^{K}(Q) \text { for } 0 \leq i \leq d-1 .
\end{aligned}
$$

We now recall few definitions. A module $M$ of dimension $r$ is said to be generalized CohenMacaulay if $\mathrm{H}_{\mathfrak{m}}^{i}(M)$ has finite length for all $0 \leq i \leq r-1$, where $\mathrm{H}_{\mathfrak{m}}^{i}(M)$ denotes the $i$-th local cohomology module of $M$ with support in $\mathfrak{m}$. For a parameter ideal $Q$, set

$I(Q ; M):=\ell_{R}(M / Q M)-e_{0}(Q, M)$ and $I(M):=\sup \{I(Q ; M): Q$ is a parameter ideal for $M\}$. It is well-known that $M$ is generalized Cohen-Macaulay if and only if $I(M)<\infty$. In this case

$$
I(M)=\sum_{i=0}^{r-1}\left(\begin{array}{c}
r-1 \\
i
\end{array}\right) \ell_{R}\left(\mathrm{H}_{\mathfrak{m}}^{i}(M)\right) .
$$

We refer [CST78] and [Tru86] for details.

Definition 2.2. (1) A parameter ideal $Q$ for $M$ is said to be standard for $M$ if $I(Q ; M)=$ $I(M)$. An ideal $I$ with $\ell_{R}(M / I M)<\infty$ is said to be $M$-standard ideal if every parameter ideal for $M$ contained in $I$ is standard for $M$.

(2) An $R$-module $M$ is said to be Buchsbaum if every parameter ideal for $M$ is standard.

In the following lemma we relate the properties of $R$ and $K$ as an $R$-module.

Lemma 2.3. Let $(R, \mathfrak{m})$ be a Noetherian local ring of dimension $d \geq 1$ and $K$ an $\mathfrak{m}$-primary ideal of R. Then

(1) $R$ is a generalized Cohen-Macaulay ring if and only if $K$ is a generalized Cohen-Macaulay $R$-module.

(2) Suppose depth $R>0$ and $K$ is a Buchsbaum $R$-module. Then $R$ is a Buchsbaum ring.

(3) If $R$ is Buchsbaum then $\mathfrak{m}$ is a Buchsbaum R-module.

Proof. (1): Consider the exact sequence

$$
0 \longrightarrow K \longrightarrow R \longrightarrow R / K \longrightarrow 0 \text {. }
$$

This induces the exact sequence

$$
0 \longrightarrow \mathrm{H}_{\mathfrak{m}}^{0}(K) \longrightarrow \mathrm{H}_{\mathfrak{m}}^{0}(R) \longrightarrow \mathrm{H}_{\mathfrak{m}}^{0}(R / K)=R / K \longrightarrow \mathrm{H}_{\mathfrak{m}}^{1}(K) \longrightarrow \mathrm{H}_{\mathfrak{m}}^{1}(R) \longrightarrow 0
$$

and isomorphisms

$$
\mathrm{H}_{\mathfrak{m}}^{i}(K) \cong \mathrm{H}_{\mathfrak{m}}^{i}(R) \text { for } 2 \leq i \leq d
$$

This shows that $\mathrm{H}_{\mathfrak{m}}^{i}(K)$ has finite length if and only if $\mathrm{H}_{\mathfrak{m}}^{i}(R)$ has finite length for $1 \leq i \leq d-1$. Hence the assertion follows.

(2): Let $Q=\left(x_{1}, \ldots, x_{d}\right)$ be an arbitrary parameter ideal of $R$. We show that $Q$ is standard for $R$. Since depth $R>0$, (2.5) gives an exact sequence

$$
0 \longrightarrow R / K \longrightarrow \mathrm{H}_{\mathfrak{m}}^{1}(K) \longrightarrow \mathrm{H}_{\mathfrak{m}}^{1}(R) \longrightarrow 0 \text {. }
$$


Thus

$$
\begin{aligned}
& \ell_{R}(R / Q K) \\
= & \ell_{R}(R / K)+\ell_{R}(K / Q K) \\
= & \ell_{R}(R / K)+\sum_{i=1}^{d-1}\left(\begin{array}{c}
d-1 \\
i
\end{array}\right) \ell_{R}\left(\mathrm{H}_{\mathfrak{m}}^{i}(K)\right)+e_{0}(Q, K) \quad \text { (as } K \text { is Buchsbaum) } \\
= & \ell_{R}(R / K)+\sum_{i=1}^{d-1}\left(\begin{array}{c}
d-1 \\
i
\end{array}\right) \ell_{R}\left(\mathrm{H}_{\mathfrak{m}}^{i}(R)\right)+(d-1) \ell_{R}(R / K)+e_{0}(Q, K) \quad \text { (from }(2.6) \text { and (2.7)) } \\
= & \left.e_{0}(Q, R)+I(R)+d \ell_{R}(R / K) \quad \text { (since } e_{0}(Q, K)=e_{0}(Q, R)\right) .
\end{aligned}
$$

Hence, by [Tru86, Corollary 4.9], $Q$ is a standard parameter ideal of $R$.

(3): Let $Q=\left(x_{1}, \ldots, x_{d}\right)$ be a parameter ideal for $\mathfrak{m}$. We have

$$
\begin{array}{rlr}
I(Q ; \mathfrak{m}) & =\ell_{R}(\mathfrak{m} / Q \mathfrak{m})-e_{0}(Q, \mathfrak{m}) & \\
& =\ell_{R}(R / Q)+\ell_{R}(Q / Q \mathfrak{m})-\ell_{R}(R / \mathfrak{m})-e_{0}(Q, R) & \\
& =I(Q ; R)+d-1 & \\
& =I(R)+d-1 & \text { (since } Q \text { is standard for } R)
\end{array}
$$

which is independent of $Q$. Hence $\mathfrak{m}$ is Buchsbaum.

\section{THE SET $\Lambda_{1}^{K}(R)$}

In this section we study the finiteness of the set $\Lambda_{1}^{K}(R)$. We give an equivalent criterion for the set $\Lambda_{1}^{K}(R)$ to be finite in an unmixed local ring (Theorem 3.2). We also consider the problem when $g_{1}^{K}(Q)$ is independent of $Q$. For $K=\mathfrak{m}$, we give a characterization for $\left|\Lambda_{1}^{K}(R)\right|=1$ in an unmixed local ring and obtain partial results for arbitrary $K$ (Theorem 3.5).

Recall that a module $M$ is said to be unmixed if $\operatorname{dim} \widehat{R} / \mathfrak{p}=\operatorname{dim} M$ for all $\mathfrak{p} \in \operatorname{Ass}_{\widehat{R}}(\widehat{M})$, where $\widehat{M}$ denotes the $m$-adic completion of $M$. In the following proposition we give bounds on $g_{1}^{K}(Q)$ in generalized Cohen-Macaulay local rings which are independent of $Q$. Consequently, we give an equivalent criterion for the finiteness of $\Lambda_{1}^{K}(R)$ in terms of $K$ in an unmixed local ring.

Proposition 3.1. Let $(R, \mathfrak{m})$ be a Noetherian local ring of dimension $d \geq 2$ and $K$ an $\mathfrak{m}$-primary ideal of $R$.

(1) Suppose $R$ is generalized Cohen-Macaulay. Then the following assertions hold.

(a) For any parameter ideal $Q$ of $R,-\sum_{i=1}^{d-1}\left(\begin{array}{c}d-2 \\ i-1\end{array}\right) \ell_{R}\left(H_{m}^{i}(R)\right)-\ell_{R}(R / K) \leq g_{1}^{K}(Q) \leq 0$. In particular, $\Lambda_{1}^{K}(R)$ is finite.

(b) If $Q$ is a standard parameter ideal for $K$, then

$$
g_{i}^{K}(Q)= \begin{cases}(-1)^{i} \sum_{j=1}^{d-i}\left(\begin{array}{c}
d-i-1 \\
j-1
\end{array}\right) \ell_{R}\left(\mathrm{H}_{\mathfrak{m}}^{j}(K)\right) & \text { if } 1 \leq i \leq d-1 \\
(-1)^{d}\left(\ell_{R}\left(\mathrm{H}_{\mathfrak{m}}^{0}(K)\right)+\ell_{R}(R / K)\right) & \text { if } i=d .\end{cases}
$$

(2) Suppose $R$ is an unmixed local ring. Then $K$ is a generalized Cohen-Macaulay (resp. Buchsbaum) $R$-module if and only if $\left|\Lambda_{1}^{K}(R)\right|<\infty\left(\right.$ resp. $\left.\left|\Lambda_{1}^{K}(R)\right|=1\right)$.

Proof. (1): Since $R$ is generalized Cohen-Macaulay, by Lemma 2.3(1), $K$ is a generalized CohenMacaulay R-module. Hence, by [GGH ${ }^{+} 15$, p. 47], $-\sum_{i=1}^{d-1}\left(\begin{array}{c}d-2 \\ i-1\end{array}\right) \ell_{R}\left(\mathrm{H}_{\mathfrak{m}}^{i}(K)\right) \leq e_{1}(Q, K) \leq 0$. 
Using (2.5) and (2.6), we get that $-\ell_{R}\left(\mathrm{H}_{\mathfrak{m}}^{1}(R)\right)-\ell_{R}(R / K) \leq-\ell_{R}\left(\mathrm{H}_{\mathfrak{m}}^{1}(K)\right)$ and $\ell_{R}\left(\mathrm{H}_{\mathfrak{m}}^{i}(R)\right)=$ $\ell_{R}\left(\mathrm{H}_{\mathfrak{m}}^{i}(K)\right)$ for all $2 \leq i \leq d-1$, respectively. Thus, $-\sum_{i=1}^{d-1}\left(\begin{array}{c}d-2 \\ i-1\end{array}\right) \ell_{R}\left(\mathrm{H}_{\mathfrak{m}}^{i}(R)\right)-\ell(R / K) \leq$ $e_{1}(Q, K) \leq 0$. Now (1a) follows from (2.1).

If $Q$ is a standard parameter ideal for $K$ then, by [Tru86, Corollary 4.2],

$$
e_{i}(Q, K)=(-1)^{i} \sum_{j=0}^{d-i}\left(\begin{array}{c}
d-i-1 \\
j-1
\end{array}\right) \ell_{R}\left(\mathrm{H}_{\mathfrak{m}}^{j}(K)\right) \text {. }
$$

Hence (1b) follows from (2.1).

(2): Since $R$ is unmixed, $K$ is an unmixed $R$-module. Also, from (2.1), $\left|\Lambda_{1}^{K}(R)\right|=\left|\Lambda_{1}(K)\right|$. Hence the result follows from $\left[\mathrm{GGH}^{+} 15\right.$, Theorem 4.5 and Theorem 5.4].

The following theorem provides an equivalent criterion for an unmixed local ring $R$ to be generalized Cohen-Macaulay in terms of the set $\Lambda_{1}^{K}(R)$.

Theorem 3.2. Let $(R, \mathfrak{m})$ be an unmixed local ring of dimension $d \geq 2$ and $K$ an $\mathfrak{m}$-primary ideal of $R$. Then the following conditions are equivalent:

(1) $R$ is generalized Cohen-Macaulay;

(2) $\Lambda_{1}^{K}(R)$ is a finite set;

(3) $\delta_{1}^{K}(R)$ is a finite set;

Proof. (1) $\Rightarrow$ (2): Follows from Proposition 3.1(1a).

(2) $\Rightarrow$ (3): Since $\delta_{1}^{K}(R) \subseteq \Lambda_{1}^{K}(R)$, the assertion follows.

$(3) \Rightarrow(1)$ : We may assume that $R$ is complete. Since $\delta_{1}^{K}(R)$ is a finite set, by (2.1), the set $S(K):=\left\{e_{1}(Q, K) \mid Q\right.$ is a parameter ideal of $R$ and $\left.Q \subseteq K\right\}$ is finite. Let $l$ be an integer such that $\mathfrak{m}^{l} \subseteq K$. Then the set

$\left\{e_{1}(Q, K) \mid Q=\left(x_{1}, \ldots, x_{d}\right) \subseteq \mathfrak{m}^{l}\right.$ is a parameter ideal of $R$ which is a $d$-sequence for $\left.K\right\} \subseteq S(K)$ is finite. Since $R$ is unmixed, $K$ is an unmixed $R$-module. Therefore by [GGH ${ }^{+} 15$, Lemma 4.1], $K$ is a generalized Cohen-Macaulay $R$-module. Hence by Proposition 2.3(1), $R$ is generalized Cohen-Macaulay.

For a finitely generated $R$-module $M$, we set $\operatorname{Assh}_{R} M=\left\{\mathfrak{p} \in \operatorname{Ass}_{R} M \mid \operatorname{dim} R / \mathfrak{p}=\operatorname{dim} M\right\}$. Let $\left(0_{M}\right)=\bigcap_{\mathfrak{p} \in \mathrm{Ass}_{R} M} M(\mathfrak{p})$ be a primary decomposition of $\left(0_{M}\right)$ in $M$, where $M(\mathfrak{p})$ is a $\mathfrak{p}$ primary submodule of $M$ for each $\mathfrak{p} \in \operatorname{Ass}_{R} M$. The $R$-submodule $U_{M}(0):=\bigcap_{\mathfrak{p} \in \operatorname{Assh}_{R} M} M(\mathfrak{p})$ is called the unmixed component of $M$.

In order to prove the next theorem we need a modified version of [GGH ${ }^{+}$15, Lemma 4.3].

Lemma 3.3. Let $(R, \mathfrak{m})$ be a Noetherian local ring and $M$ a finitely generated $R$-module with $\operatorname{dim} M=$ $r \geq 2$. Let $K$ be an $\mathfrak{m}$-primary ideal of $R$. Assume that there exists an integer $t \geq 0$ such that $e_{1}(Q, M) \geq-t$ for every parameter ideal $Q \subseteq K$ for $M$. Then $\operatorname{dim} U_{M}(0) \leq r-2$.

Proof. Let $U=U_{M}(0)$ and $T=M / U$. Since $U_{\mathfrak{p}}=0$ for all $\mathfrak{p} \in \operatorname{Assh}_{R}(M), \operatorname{dim} U \leq r-1$. Suppose $\operatorname{dim} U=r-1$. Choose a system of parameters $\left(x_{1}, \ldots, x_{r}\right)$ for $M$ such that $x_{r} U=0$. Since $\mathfrak{m}^{l} \subseteq K$ for some integer $l \geq 1, Q=\left(x_{1}^{s}, \ldots, x_{r}^{s}\right) \subseteq K$ for all $s \geq l$. Let $s>\max \{l, t\}$. Consider the exact sequence

$$
0 \longrightarrow U /\left(Q^{n+1} M \cap U\right) \longrightarrow M / Q^{n+1} M \longrightarrow T / Q^{n+1} T \longrightarrow 0 .
$$

This gives

$$
\ell_{R}\left(M / Q^{n+1} M\right)=\ell_{R}\left(T / Q^{n+1} T\right)+\ell_{R}\left(U /\left(Q^{n+1} M \cap U\right)\right)
$$


By Artin-Rees Lemma there exists an integer $k \geq 0$ such that $Q^{n} M \cap U=Q^{n-k}\left(Q^{k} M \cap U\right)$ for all $n \geq k$. Let $U^{\prime}=Q^{k} M \cap U$ and $q=\left(x_{1}^{s}, \ldots, x_{r-1}^{s}\right)$. Since $Q^{n-k} U^{\prime}=q^{n-k} U^{\prime}$ for all $n \geq k$, we get

$$
\ell_{R}\left(M / Q^{n+1} M\right)=\ell_{R}\left(T / Q^{n+1} T\right)+\ell_{R}\left(U^{\prime} / q^{n+1-k} U^{\prime}\right)+\ell_{R}\left(U / U^{\prime}\right) \text { for all } n \geq k .
$$

This implies that $-t \leq e_{1}(Q, M)=e_{1}(Q, T)-e_{0}\left(q, U^{\prime}\right)$. Since $e_{0}\left(q, U^{\prime}\right)=e_{0}(q, U)$ and $e_{1}(Q, T) \leq$ 0 by [MSV11, Theorem 3.6], we get

$$
s \leq s^{r-1} e_{0}\left(\left(x_{1}, \ldots, x_{r-1}\right), U\right)=e_{0}(q, U)=e_{1}(Q, T)-e_{1}(Q, M) \leq t,
$$

which is a contradiction. Thus $\operatorname{dim} U \leq r-2$.

In the following theorem we give equivalent conditions for the finiteness of the set $\Lambda_{1}^{K}(R)$ in any Noetherian local ring.

Theorem 3.4. Let $(R, \mathfrak{m})$ be a Noetherian local ring of dimension $d \geq 2$. We set $U=U_{\widehat{R}}(0)$. Then the following conditions are equivalent:

(1) $\operatorname{dim}_{\widehat{R}} U \leq d-2$ and $\widehat{R} / U$ is a generalized Cohen-Macaulay ring;

(2) $\Lambda_{1}^{K}(R)$ is a finite set;

(3) $\delta_{1}^{K}(R)$ is a finite set.

When this is the case, we have

$$
-\sum_{i=1}^{d-1}\left(\begin{array}{c}
d-2 \\
i-1
\end{array}\right) \ell_{R}\left(\mathrm{H}_{\mathfrak{m}}^{i}(\widehat{R} / U)\right)-\ell_{R}(R / K) \leq g_{1}^{K}(Q) \leq 0 .
$$

for every parameter ideal $Q$ of $R$.

Proof. We may assume that $R$ is complete.

$(1) \Rightarrow(2)$ : Since $R / U$ is a generalized Cohen-Macaulay ring, by Proposition 3.1(1a), the set $\Lambda_{1}^{K R / U}(R / U)$ is finite. Since $g_{1}^{K}(R)=g_{1}^{K R / U}(R / U)$, by [Sal16, Lemma 3.6], the set $\Lambda_{1}^{K}(R)$ is finite. (Note that we do not need $Q \subseteq K$ in [Sal16, Lemma 3.6].)

(2) $\Rightarrow$ (3): Since $\delta_{1}^{K}(R) \subseteq \Lambda_{1}^{K}(R)$, the assertion follows.

$(3) \Rightarrow(1)$ : From (2.1), $e_{1}(Q, K)=g_{1}^{K}(Q)$. Thus $\delta_{1}^{K}(R)$ is finite implies that there exists an integer $t \geq 0$ such that $e_{1}(Q, K) \geq-t$ for every parameter ideal $Q \subseteq K$. Hence, by Lemma 3.3, $\operatorname{dim} U_{K}(0) \leq d-2$. Note that $U \cap K=U_{K}(0)$. Since $\operatorname{dim} U=\max \{\operatorname{dim}(U \cap K), \operatorname{dim}(U /(U \cap$ $K))\}$ and $\operatorname{dim}(U /(U \cap K))=0, \operatorname{dim} U=\operatorname{dim}(U \cap K)=\operatorname{dim}\left(U_{K}(0)\right) \leq d-2$. Hence, by [Sal16, Lemma 3.6], $g_{1}^{K}(Q)=g_{1}^{K R / U}(Q R / U)$. Using [GGH ${ }^{+} 15$, Remark 4.4] we conclude that $\delta_{1}^{K R / U}(R / U)$ is finite. Hence, by Theorem 3.2, $R / U$ is generalized Cohen-Macaulay.

The last assertion follows from Proposition 3.1(1a).

In the following theorem we give a sufficient condition for $R$ to be Buchsbaum.

Theorem 3.5. Let $(R, \mathfrak{m})$ be a Noetherian local ring of dimension $d \geq 2$ and $K$ an $\mathfrak{m}$-primary ideal of $R$. Then the following assertions hold.

(1) Suppose $R$ is unmixed and $\left|\Lambda_{1}^{K}(R)\right|=1$. Then $R$ is Buchsbaum. Further, $\left|\Lambda_{i}^{K}(R)\right|=1$ for all $1 \leq i \leq d$.

(2) If $R$ is Buchsbaum then $\left|\Lambda_{1}^{\mathfrak{m}}(R)\right|=1$.

Proof. (1): By Proposition 3.1(2), we get that $K$ is a Buchsbaum $R$-module. Hence by Lemma 2.3(2), $R$ is a Buchsbaum ring. Since every parameter ideal of $R$ is standard for $K$, by Proposition 3.1(1b), $\left|\Lambda_{i}^{K}(R)\right|=1$ for all $1 \leq i \leq d$.

(2): By Lemma 2.3(3), $\mathfrak{m}$ is a Buchsbaum $R$-module. Thus every parameter ideal $Q$ of $R$ is standard for $\mathfrak{m}$. Now by Proposition 3.1(1b), $\left|\Lambda_{1}^{\mathfrak{m}}(R)\right|=1$. 
Theorem 3.6. Let $(R, \mathfrak{m})$ be a Noetherian local ring of dimension $d \geq 2$. Let $U=U_{\widehat{R}}(0)$. Then the following conditions are equivalent:

(1) $\operatorname{dim}_{\widehat{R}} U \leq d-2$ and $\widehat{R} / U$ is Buchsbaum;

(2) $\left|\Lambda_{1}^{\mathfrak{m}}(R)\right|=1$.

Proof. We may assume that $R$ is complete.

$(1) \Rightarrow$ (2): By [Sal16, Lemma 3.6] and Theorem 3.5(2), we get $\left|\Lambda_{1}^{\mathfrak{m}}(R)\right|=\left|\Lambda_{1}^{\mathfrak{m}}(R / U)\right|=1$.

$(2) \Rightarrow(1)$ : Since $\left|\Lambda_{1}^{\mathfrak{m}}(R)\right|=1$, by $(2.1),\left|\Lambda_{1}(\mathfrak{m})\right|=1$. Since $U_{\mathfrak{m}}(0)=U$, by [GGH ${ }^{+} 15$, Theorem 5.5], $\operatorname{dim} U=\operatorname{dim} U_{\mathfrak{m}}(0) \leq d-2$. Thus $g_{1}^{\mathfrak{m}}(Q)=g_{1}^{\mathfrak{m} R / U}(Q R / U)$ by [Sal16, Lemma 3.6]. Hence $\left|\Lambda_{1}^{\mathfrak{m}}(R / U)\right|=\left|\Lambda_{1}^{\mathfrak{m}}(R)\right|=1$. Therefore, by Theorem 3.5(1), $R / U$ is Buchsbaum.

We discuss below that for an arbitrary m-primary ideal $K$ in a Buchsbaum local ring $R$, $\Lambda_{1}^{K}(R)$ need not be singleton.

Discussion 3.7. Suppose $(R, \mathfrak{m})$ is a Buchsbaum local ring of dimension $d \geq 2$ and $K$ is an $\mathfrak{m}$-primary ideal of $R$. Suppose $\left|\Lambda_{1}^{K}(R)\right|=1$. Then, from (2.1), $\left|\Lambda_{1}(K)\right|=1$. Further assume that $R$ is unmixed. Then, by [GGH ${ }^{+} 15$, Theorem 5.4], $K$ is a Buchsbaum $R$-module. Let $Q$ be an arbitrary parameter ideal of $R$. Since

$$
\ell_{R}\left(R / Q^{n+1} K\right)=\ell_{R}(R / K)+\ell_{R}\left(K / Q^{n+1} K\right) \text { for all } n
$$

and $Q$ is standard for $K$, using [Tru86, Corollary 4.2], we get

$\ell_{R}\left(R / Q^{n+1} K\right)=\ell_{R}(R / K)+\left(\begin{array}{c}n+d \\ d\end{array}\right) e_{0}(Q, K)+\sum_{i=1}^{d} \sum_{j=0}^{d-i}\left(\begin{array}{c}n+d-i \\ d-i\end{array}\right)\left(\begin{array}{c}d-i-1 \\ j-1\end{array}\right) \ell_{R}\left(\mathrm{H}_{\mathfrak{m}}^{j}(K)\right)$

for all $n \geq 0$. Putting $n=0$ and using (2.5) and (2.6), we get

$$
\ell_{R}(R / Q K)=e_{0}(Q, R)+I(R)+d \ell(R / K) \text {. }
$$

Also,

$$
\begin{aligned}
\ell_{R}(R / Q K) & =\ell_{R}(R / Q)+\ell_{R}(Q / Q K) \\
& =e_{0}(Q, R)+I(R)+\ell_{R}(Q / Q K) .
\end{aligned}
$$

Comparing (3.1) and (3.2), we get $\ell_{R}(Q / Q K)=d \ell_{R}(R / K)$ for every parameter ideal $Q$ of $R$. This need not be true even in regular local rings.

However, we expect that $\left|\delta_{1}^{K}(R)\right|=1$ for an arbitrary m-primary ideal $K$ in a Buchsbaum local ring. We have neither a proof nor a counter-example for this statement.

Remark 3.8. (1) Suppose $R$ is a generalized Cohen-Macaulay local ring. Then, by $\left[\mathrm{GGH}^{+} 15\right.$, Section 4], $\Lambda_{1}(R)$ is finite. By Proposition 3.1(1), $\Lambda_{1}^{K}(R)$ is finite. Hence, from (2.3), the set $\left\{f_{0}^{K}(Q) \mid Q\right.$ is a parameter ideal of $\left.R\right\}$ is finite.

(2) Suppose $R$ is Buchsbaum. Then, by [GGH ${ }^{+} 15$, Section 5], $\Lambda_{1}(R)$ is singleton. By Theorem 3.5(2), $\left|\Lambda_{1}^{\mathfrak{m}}(R)\right|=1$. Hence, from (2.3), the set $\left\{f_{0}^{\mathfrak{m}}(Q) \mid Q\right.$ is a parameter ideal of $\left.R\right\}$ is singleton.

\section{THE SET $\Lambda_{i}^{K}(R)$}

In this section we give a necessary and sufficient condition for $\Lambda_{i}^{K}(R)$ to be finite for all $1 \leq$ $i \leq d$ (Theorem 4.9). For this purpose we improve a result of Goto and Ozeki [GO11, Theorem 1.1] and generalize it for modules (Theorem 4.7). We also obtain an equivalent criterion for $\left|\Lambda_{i}(M)\right|=1$ for all $1 \leq i \leq r-\operatorname{depth} M$ (Theorem 4.10). As a consequence we obtain a necessary condition for $\left|\Lambda_{i}^{K}(R)\right|=1$ for all $1 \leq i \leq d-1$ (Theorem 4.11).

We need few lemmas in order to prove the finiteness of $\Lambda_{i}^{K}(R)$ in a generalized CohenMacaulay local ring. First we recall the following lemma from [Tru86]. 
Lemma 4.1. [Tru86, Lemma 1.7] Let $M$ be a generalized Cohen-Macaulay module of dimension $r$ and $Q=\left(x_{1}, \ldots, x_{r}\right)$ a parameter ideal for $M$. Then $I\left(M / x_{1} M\right) \leq I(M)$.

In the next lemma we give a bound on the function $\ell_{R}\left(M / Q^{n+1} M\right)$ in terms of $e_{0}(Q, M)$ and $I(M)$ for a generalized Cohen-Macaulay module. A similar upper bound is given for $\ell_{R}\left(R / Q^{n+1}\right)$ in [LT06, Lemma 1.1]. A better lower bound is given for $\ell_{R}\left(R / Q^{n+1}\right)$ in terms of $e_{0}(Q, R)$ in [HH11, Theorem 1.1].

Lemma 4.2. Let $M$ be a generalized Cohen-Macaulay module of dimension $r>0$ and $Q$ a parameter ideal for $M$. Then for all $n \geq 0$,

$$
-r\left(\begin{array}{c}
n+r-1 \\
r-1
\end{array}\right) I(M) \leq \ell_{R}\left(M / Q^{n+1} M\right)-e_{0}(Q, M)\left(\begin{array}{c}
n+r \\
r
\end{array}\right) \leq\left(\begin{array}{c}
n+r-1 \\
r-1
\end{array}\right) I(M) .
$$

Proof. We apply induction on $r$. Let $r=1$. Set $W:=\mathrm{H}_{\mathfrak{m}}^{0}(M)$ and $M^{\prime}:=M / \mathrm{H}_{\mathfrak{m}}^{0}(M)$. Then $M^{\prime}$ is a Cohen-Macaulay $R$-module and $e_{0}\left(Q, M^{\prime}\right)=e_{0}(Q, M)$. Hence for all $n \geq 0$,

$$
\begin{aligned}
\ell_{R}\left(M / Q^{n+1} M\right) & =\ell_{R}\left(M^{\prime} / Q^{n+1} M^{\prime}\right)+\ell_{R}\left(W /\left(W \cap Q^{n+1} M\right)\right) \\
& =e_{0}(Q, M)(n+1)+\ell_{R}\left(W /\left(W \cap Q^{n+1} M\right)\right) .
\end{aligned}
$$

Therefore for all $n \geq 0$,

$$
0 \leq \ell_{R}\left(M / Q^{n+1} M\right)-e_{0}(Q, M)(n+1) \leq \ell_{R}(W)=I(M)
$$

Thus the result is true for $r=1$. Now let $r>1$ and $Q=\left(x_{1}, \ldots, x_{r}\right)$ be a parameter ideal for $M$. We put $\bar{M}=M / x_{1} M$. Since $M$ is a generalized Cohen-Macaulay module, by [CLT15, Claim 1 in p. 351], we have

$$
0 \leq \ell_{R}\left(\left(Q^{n+1} M: x_{1}\right) / Q^{n} M\right) \leq\left(\begin{array}{c}
n+r-2 \\
r-2
\end{array}\right) I(M) \text { for all } n \geq 0 .
$$

By [Tru86, Lemma 1.2], $\operatorname{dim} R / \mathfrak{p}=r-i$ for all $\mathfrak{p} \in \operatorname{Ass}\left(M /\left(x_{1}, \ldots, x_{i}\right) M\right) \backslash\{\mathfrak{m}\}$ and $i=$ $1, \ldots, r-1$. Thus, using [AB58, Corollary 4.8], we get

$$
e_{0}(Q, \bar{M})=\ell_{R}(M / Q M)-\ell_{R}\left(\left(x_{1}, \ldots, x_{r-1}\right) M: x_{r} / x_{r-1} M\right)=e_{0}(Q, M) .
$$

By Lemma 4.1, $I(\bar{M}) \leq I(M)$. Hence applying induction hypothesis, we get

$$
-(r-1)\left(\begin{array}{c}
n+r-2 \\
r-2
\end{array}\right) I(M) \leq \ell_{R}\left(\bar{M} / Q^{n+1} \bar{M}\right)-e_{0}(Q, M)\left(\begin{array}{c}
n+r-1 \\
r-1
\end{array}\right) \leq\left(\begin{array}{c}
n+r-2 \\
r-2
\end{array}\right) I(M)
$$

for all $n \geq 0$. Considering the exact sequence

$$
0 \longrightarrow\left(Q^{t+1} M: x_{1}\right) / Q^{t} M \longrightarrow M / Q^{t} M \stackrel{x_{1}}{\longrightarrow} M / Q^{t+1} M \longrightarrow \bar{M} / Q^{t+1} \bar{M} \longrightarrow 0,
$$

we get

$$
\ell_{R}\left(Q^{t} M / Q^{t+1} M\right)=\ell_{R}\left(\bar{M} / Q^{t+1} \bar{M}\right)-\ell_{R}\left(\left(Q^{t+1} M: x_{1}\right) / Q^{t} M\right) \text { for all } t \geq 0 .
$$

Hence, using (4.1) and (4.2), we get

$$
-r\left(\begin{array}{c}
t+r-2 \\
r-2
\end{array}\right) I(M) \leq \ell_{R}\left(Q^{t} M / Q^{t+1} M\right)-e_{0}(Q, M)\left(\begin{array}{c}
t+r-1 \\
r-1
\end{array}\right) \leq\left(\begin{array}{c}
t+r-2 \\
r-2
\end{array}\right) I(M)
$$

Since $\ell_{R}\left(M / Q^{n+1} M\right)=\sum_{t=0}^{n} \ell_{R}\left(Q^{t} M / Q^{t+1} M\right)$, using (4.4), we get

$$
-r\left(\begin{array}{c}
n+r-1 \\
r-1
\end{array}\right) I(M) \leq \ell_{R}\left(M / Q^{n+1} M\right)-e_{0}(Q, M)\left(\begin{array}{c}
n+r \\
r
\end{array}\right) \leq\left(\begin{array}{c}
n+r-1 \\
r-1
\end{array}\right) I(M) .
$$


Let $G_{Q}(M)=\bigoplus_{n \geq 0} Q^{n} M / Q^{n+1} M$ be the associated graded module of $M$ with respect to $Q$. Let $\mathcal{M}=\bigoplus_{n \geq 1}\left[G_{Q}(R)\right]_{n}$ and

$$
a_{i}\left(G_{Q}(M)\right)=\sup \left\{n \in \mathbb{Z}:\left[H_{\mathcal{M}}^{i}\left(G_{Q}(M)\right)\right]_{n} \neq 0\right\} .
$$

Recall that

$$
\operatorname{reg}\left(G_{Q}(M)\right)=\sup \left\{a_{i}\left(G_{Q}(M)\right)+i: i \in \mathbb{Z}\right\}
$$

is the Castelnuovo-Mumford regularity of the graded module $G_{Q}(M)$. We need the following lemma in order to obtain uniform bounds on the coefficients $e_{i}(Q, M)$ in terms of $\operatorname{reg}\left(G_{Q}(M)\right)$. We skip the proof of this as it is similar to [GO11, Lemma 2.3].

Lemma 4.3. Let $M$ be a finitely generated module of dimension $r>0$ and $Q$ a parameter ideal for $M$.

(1) Let $M^{\prime}=M / \mathrm{H}_{\mathfrak{m}}^{0}(M)$. Then $\operatorname{reg}\left(G_{Q}(M)\right) \geq \operatorname{reg}\left(G_{Q}\left(M^{\prime}\right)\right)$.

(2) Assume that $r \geq 2$ and $x \in Q$ is superficial for $M$ with respect to $Q$. Let $\bar{Q}=Q /(x)$ in $\bar{R}=R /(x)$ and $\bar{M}=M / x M$. Then $\operatorname{reg}\left(G_{Q}(M)\right) \geq \operatorname{reg}\left(G_{\bar{Q}}(\bar{M})\right)$.

We use a method similar to [GO11, Theorem 2.2] to prove the following theorem.

Theorem 4.4. Let $M$ be a generalized Cohen-Macaulay module of dimension $r>0$ and $Q$ a parameter ideal for $M$. Put $\kappa=\operatorname{reg}\left(G_{Q}(M)\right)$. Then

(1) $\left|e_{1}(Q, M)\right| \leq I(M)$.

(2) $\left|e_{i}(Q, M)\right| \leq(r+1) \cdot 2^{i-2}(\kappa+1)^{i-1} I(M)$ for $2 \leq i \leq r$.

Proof. We may assume that the residue field $R / \mathfrak{m}$ is infinite. We use induction on $r$. Let $r=1$. Then by [MSV11, Proposition 3.1], $e_{1}(Q, M)=-\ell_{R}\left(\mathrm{H}_{\mathfrak{m}}^{0}(M)\right)$ for all parameter ideals $Q$ for $M$. Hence $\left|e_{1}(Q, M)\right|=\ell_{R}\left(\mathrm{H}_{\mathfrak{m}}^{0}(M)\right)=I(M)$. Thus the assertion is true in this case.

Let $r \geq 2$. We may assume that $\operatorname{depth} M>0$. In fact, let $M^{\prime}=M / \mathrm{H}_{\mathfrak{m}}^{0}(M)$ and assume that the assertion holds for $M^{\prime}$. Set $\kappa^{\prime}=\operatorname{reg}\left(G_{Q}\left(M^{\prime}\right)\right)$. By [RV10, Proposition 2.3],

$$
e_{i}(Q, M)= \begin{cases}e_{i}\left(Q, M^{\prime}\right) & \text { if } i \neq r \\ e_{r}\left(Q, M^{\prime}\right)+(-1)^{r} \ell_{R}\left(\mathrm{H}_{\mathfrak{m}}^{0}(M)\right) & \text { if } i=r\end{cases}
$$

Hence

$$
\left|e_{1}(Q, M)\right| \leq\left|e_{1}\left(Q, M^{\prime}\right)\right|+\ell_{R}\left(H_{\mathfrak{m}}^{0}(M)\right) \leq I\left(M^{\prime}\right)+\ell_{R}\left(H_{\mathfrak{m}}^{0}(M)\right)=I(M)
$$

and for $2 \leq i \leq r$,

$$
\begin{aligned}
& \left|e_{i}(Q, M)\right| \leq\left|e_{i}\left(Q, M^{\prime}\right)\right|+\ell_{R}\left(\mathrm{H}_{\mathfrak{m}}^{0}(M)\right) \\
& \leq(r+1) \cdot 2^{i-2}\left(\kappa^{\prime}+1\right)^{i-1} I\left(M^{\prime}\right)+\ell_{R}\left(\mathrm{H}_{\mathfrak{m}}^{0}(M)\right) \\
& \leq(r+1) \cdot 2^{i-2}\left(\kappa^{\prime}+1\right)^{i-1}\left(\sum_{i=1}^{r-1}\left(\begin{array}{c}
r-1 \\
i
\end{array}\right) \ell_{R}\left(\mathrm{H}_{\mathfrak{m}}^{i}\left(M^{\prime}\right)\right)+\ell_{R}\left(\mathrm{H}_{\mathfrak{m}}^{0}(M)\right)\right) \\
& \leq(r+1) \cdot 2^{i-2}(\kappa+1)^{i-1}\left(\sum_{i=0}^{r-1}\left(\begin{array}{c}
r-1 \\
i
\end{array}\right) \ell_{R}\left(\mathrm{H}_{\mathfrak{m}}^{i}(M)\right)\right) \quad \text { [by Lemma 4.3(1)] } \\
& =(r+1) \cdot 2^{i-2}(\kappa+1)^{i-1} I(M) .
\end{aligned}
$$

Let $Q=\left(x_{1}, \ldots, x_{r}\right)$ be such that $x_{1}$ is superficial for $M$ with respect to $Q$. Put $\bar{M}=M / x_{1} M$ and $\bar{\kappa}=\operatorname{reg}\left(G_{Q}(\bar{M})\right.$. Then, using induction hypothesis and Lemmas 4.1 and 4.3(2), we get

$$
\left|e_{1}(Q, M)\right|=\left|e_{1}(Q, \bar{M})\right| \leq I(\bar{M}) \leq I(M)
$$


and for $2 \leq i \leq r-1$,

(4.7) $\left|e_{i}(Q, M)\right|=\left|e_{i}(Q, \bar{M})\right| \leq r \cdot 2^{i-2}(\bar{\kappa}+1)^{i-1} I(\bar{M}) \leq(r+1) \cdot 2^{i-2}(\kappa+1)^{i-1} I(M)$.

Let $i=r$. By [BH93, Theorem 4.4.3], for all $t>\kappa$

$$
\ell_{R}\left(Q^{t} M / Q^{t+1} M\right)=\sum_{i=0}^{r-1}(-1)^{i} e_{i}(Q, M)\left(\begin{array}{c}
t+r-1-i \\
r-1-i
\end{array}\right)
$$

and for all $t \geq \bar{\kappa}$,

$$
\ell_{R}\left(\bar{M} / Q^{t+1} \bar{M}\right)=\sum_{i=0}^{r-1}(-1)^{i} e_{i}(Q, \bar{M})\left(\begin{array}{c}
t+r-1-i \\
r-1-i
\end{array}\right) .
$$

Since $\kappa \geq \bar{\kappa}$ by Lemma 4.3(2), we get $\ell_{R}\left(Q^{t} M / Q^{t+1} M\right)=\ell_{R}\left(\bar{M} / Q^{t+1} \bar{M}\right)$ for all $t>\kappa$. Hence, using (4.3), we get $\left(Q^{t+1} M: x_{1}\right)=Q^{t} M$ for all $t>\kappa$. Since $\ell_{R}\left(M / Q^{n+1} M\right)=$ $\sum_{i=0}^{r}(-1)^{i} e_{i}(Q, M)\left(\begin{array}{c}n+r-i \\ r-i\end{array}\right)$ for all $n \geq \kappa$ by [BH93, Theorem 4.4.3], we get

$$
\begin{aligned}
& (-1)^{r} e_{r}(Q, M) \\
= & \ell_{R}\left(M / Q^{n+1} M\right)-\sum_{i=0}^{r-1}(-1)^{i} e_{i}(Q, M)\left(\begin{array}{c}
n+r-i \\
r-i
\end{array}\right) \\
= & \sum_{t=0}^{n} \ell_{R}\left(Q^{t} M / Q^{t+1} M\right)-\sum_{i=0}^{r-1}(-1)^{i} e_{i}(Q, M)\left(\begin{array}{c}
n+r-i \\
r-i
\end{array}\right) \\
= & \sum_{t=0}^{n} \ell_{R}\left(\bar{M} / Q^{t+1} \bar{M}\right)-\sum_{t=0}^{n} \ell_{R}\left(\left(Q^{t+1} M: x_{1}\right) / Q^{t} M\right)-\sum_{t=0}^{n} \sum_{i=0}^{r-1}(-1)^{i} e_{i}(Q, \bar{M})\left(\begin{array}{c}
t+r-1-i \\
r-1-i
\end{array}\right) \\
= & \sum_{t=0}^{n}\left(\ell_{R}\left(\bar{M} / Q^{t+1} \bar{M}\right)-\sum_{i=0}^{r-1}(-1)^{i} e_{i}(Q, \bar{M})\left(\begin{array}{c}
t+r-3)] \\
r-1-i
\end{array}\right)\right)-\sum_{t=0}^{\kappa} \ell_{R}\left(\left(Q^{t+1} M: x_{1}\right) / Q^{t} M\right) \\
= & \sum_{t=0}^{\bar{\kappa}}\left(\ell_{R}\left(\bar{M} / Q^{t+1} \bar{M}\right)-\sum_{i=0}^{r-1}(-1)^{i} e_{i}(Q, \bar{M})\left(\begin{array}{c}
t+r-1-i \\
r-1-i
\end{array}\right)\right)-\sum_{t=0}^{\kappa} \ell_{R}\left(\left(Q^{t+1} M: x_{1}\right) / Q^{t} M\right) \\
= & \sum_{t=0}^{\bar{\kappa}}\left(\ell_{R}\left(\bar{M} / Q^{t+1} \bar{M}\right)-e_{0}(Q, \bar{M})\left(\begin{array}{c}
t+r-1 \\
r-1
\end{array}\right)\right)-\sum_{t=0}^{\bar{\kappa}} \sum_{i=1}^{r-1}(-1)^{i} e_{i}(Q, \bar{M})\left(\begin{array}{c}
t+r-1-i \\
r-1-i
\end{array}\right) \\
& -\sum_{t=0}^{\kappa} \ell_{R}\left(\left(Q^{t+1} M: x_{1}\right) / Q^{t} M\right) .
\end{aligned}
$$

This implies that

$$
\begin{aligned}
& \left|e_{r}(Q, M)\right| \\
\leq & \sum_{t=0}^{\bar{\kappa}}\left|\ell_{R}\left(\bar{M} / Q^{t+1} \bar{M}\right)-e_{0}(Q, \bar{M})\left(\begin{array}{c}
t+r-1 \\
r-1
\end{array}\right)\right|+\sum_{t=0}^{\bar{\kappa}}\left|e_{1}(Q, M)\right|\left(\begin{array}{c}
t+r-2 \\
r-2
\end{array}\right) \\
& +\sum_{t=0}^{\bar{\kappa}} \sum_{i=2}^{r-1}\left|e_{i}(Q, M)\right|\left(\begin{array}{c}
t+r-1-i \\
r-1-i
\end{array}\right)+\sum_{t=0}^{\kappa} \ell_{R}\left(\left(Q^{t+1} M: x_{1}\right) / Q^{t} M\right) \\
\leq & \sum_{t=0}^{\bar{\kappa}}(r-1)\left(\begin{array}{c}
t+r-2 \\
r-2
\end{array}\right) I(\bar{M})+\sum_{t=0}^{\bar{\kappa}}\left(\begin{array}{c}
t+r-2 \\
r-2
\end{array}\right) I(M)
\end{aligned}
$$




$$
\begin{aligned}
& +\sum_{t=0}^{\bar{\kappa}} \sum_{i=2}^{r-1}(r+1) \cdot 2^{i-2}(\kappa+1)^{i-1}\left(\begin{array}{c}
t+r-1-i \\
r-1-i
\end{array}\right) I(M)+\sum_{t=0}^{\kappa}\left(\begin{array}{c}
t+r-2 \\
r-2
\end{array}\right) I(M) \\
= & (r-1)\left(\begin{array}{c}
\bar{\kappa}+r-1 \\
r-1
\end{array}\right) I(\bar{M})+\left(\begin{array}{c}
\bar{\kappa}+r-1 \\
r-1
\end{array}\right) I(M) \\
& +\sum_{i=2}^{r-1}(r+1) \cdot 2^{i-2}(\kappa+1)^{i-1}\left(\begin{array}{c}
\bar{\kappa}+r-i \\
r-i
\end{array}\right) I(M)+\left(\begin{array}{c}
\kappa+r-1 \\
r-1
\end{array}\right) I(M) \\
\leq & (r+1)\left(\begin{array}{c}
\kappa+r-1 \\
r-1
\end{array}\right) I(M)+\sum_{i=2}^{r-1}(r+1) \cdot 2^{i-2}(\kappa+1)^{i-1}\left(\begin{array}{c}
\kappa+r-i \\
r-i
\end{array}\right) I(M) \\
\leq & (r+1)(\kappa+1)^{r-1} I(M)+\sum_{i=2}^{r-1}(r+1) \cdot 2^{i-2}(\kappa+1)^{r-1} I(M) \\
= & (r+1)(\kappa+1)^{r-1} I(M)\left(1+\sum_{i=2}^{r-1} 2^{i-2}\right) \quad\left[\text { since }\left(\begin{array}{c}
m+n \\
n
\end{array}\right) \leq(m+1)^{n} \text { for all integers } n \geq 0\right] \\
= & (r+1) \cdot 2^{r-2}(\kappa+1)^{r-1} I(M) \quad\left[\begin{array}{c}
r-1 \\
i=2
\end{array} 2^{i-2}=2^{r-2}-1\right] .
\end{aligned}
$$

In the following lemma we give a necessary condition for the finiteness of the set $\Lambda_{i}(M)$ for all $1 \leq i \leq k$, where $k$ is a fixed integer such that $1 \leq k \leq r$. The proof given here is motivated by [GO11, Theorem 1.1].

Lemma 4.5. Let $(R, \mathfrak{m})$ be a Noetherian local ring, $K$ an $\mathfrak{m}$-primary ideal of $R$ and $M$ a finitely generated $R$-module of dimension $r \geq 2$. For a fixed $1 \leq k \leq r$, assume that

$$
\left\{e_{i}(Q, M): Q \text { is a parameter ideal for } M \text { and } Q \subseteq K\right\}
$$

is a finite set for all $1 \leq i \leq k$. Then $\ell_{R}\left(\mathrm{H}_{\mathfrak{m}}^{r-i}(M)\right)<\infty$ for all $1 \leq i \leq k$.

In particular, if $\Lambda_{i}(M)$ is finite for all $1 \leq i \leq k$, then $\ell_{R}\left(\mathrm{H}_{\mathfrak{m}}^{r-i}(M)\right)<\infty$ for all $1 \leq i \leq k$.

Proof. We may assume that $R$ is complete. Let $l$ be an integer such that $\mathfrak{m}^{l} \subseteq K$. Let $U=U_{M}(0)$ and $N=M / U$. If $U=0$ then $M$ is unmixed and the set $\left\{e_{1}(Q, M): Q=\left(x_{1}, \ldots, x_{r}\right) \subseteq\right.$ $\mathfrak{m}^{l}$ and $x_{1}, \ldots, x_{r}$ is a $d$-sequence for $\left.M\right\}$ is finite. Hence by [GGH ${ }^{+} 15$, Lemma 4.1], $M$ is a generalized Cohen-Macaulay module. Thus $\ell_{R}\left(\mathrm{H}_{\mathfrak{m}}^{r-i}(R)\right)<\infty$ for all $1 \leq i \leq r$.

Assume that $U \neq 0$. By Lemma 3.3, $\operatorname{dim} U \leq r-2$. Hence by [GGH ${ }^{+} 15$, Lemma 3.3], $e_{1}(Q, M)=e_{1}(Q, N)$. Thus the set $\left\{e_{1}(Q, N): Q\right.$ is a parameter ideal for $M$ and $\left.Q \subseteq K\right\}$ is finite. By $\left[\mathrm{GGH}^{+} 15\right.$, Remark 4.4], the $\operatorname{set}\left\{e_{1}(Q, N): Q\right.$ is a parameter ideal for $N$ and $\left.Q \subseteq K\right\}$ is also finite. Hence, by $U=0$ case, $N$ is generalized Cohen-Macaulay. We now show that $t:=\operatorname{dim} U \leq r-(k+1)$. We may assume that $t \geq 1$. Let $x_{1}, \ldots, x_{r}$ be a system of parameters for $M$ such that $\left(x_{t+1}, \ldots, x_{r}\right) U=0$. Since $N$ is a generalized Cohen-Macaulay module, by [Tru86, Lemma 1.5], there exists an integer $l_{1} \geq 1$ such that $\mathfrak{m}^{l_{1}}$ is a standard ideal for $N$. Let $l_{0}=\max \left\{l_{1}, l\right\}$. Then $\mathfrak{m}^{l_{0}} \subseteq K$ is a standard ideal. Let $n \geq l_{0}$ and $Q=\left(x_{1}^{n}, \ldots, x_{r}^{n}\right)$. Then by 
[Tru86, Corollary 4.2],

$$
e_{r-t}(Q, N)=(-1)^{r-t} \sum_{j=1}^{t}\left(\begin{array}{l}
t-1 \\
j-1
\end{array}\right) \ell_{R}\left(\mathrm{H}_{\mathfrak{m}}^{j}(N)\right) .
$$

We have

$$
\ell_{R}\left(M / Q^{n+1} M\right)=\ell_{R}\left(N / Q^{n+1} N\right)+\ell_{R}\left(U /\left(Q^{n+1} M \cap U\right)\right) \text { for all } n \geq 0 .
$$

Since the filtration $\left\{Q^{n+1} M \cap U\right\}$ is a good $Q$-filtration of $U$ (see [RV10, page 1] for the definition of good $Q$-filtration),

$$
\ell_{R}\left(U /\left(Q^{n+1} M \cap U\right)\right)=\sum_{i=0}^{t}(-1)^{i} s_{i}(Q, U)\left(\begin{array}{c}
n+t-i \\
t-i
\end{array}\right)
$$

for some integers $s_{i}(Q, U)$ with $s_{0}(Q, U)=e_{0}(Q, U)$. This implies that for $n \gg 0$,

$$
\ell_{R}\left(M / Q^{n+1} M\right)=\sum_{i=0}^{r}(-1)^{i} e_{i}(Q, N)\left(\begin{array}{c}
n+r-i \\
r-i
\end{array}\right)+\sum_{i=0}^{t}(-1)^{i} s_{i}(Q, U)\left(\begin{array}{c}
n+t-i \\
t-i
\end{array}\right) .
$$

Therefore for $n \geq l_{0}$,

$$
\begin{aligned}
(-1)^{r-t} e_{r-t}(Q, M) & =(-1)^{r-t} e_{r-t}(Q, N)+e_{0}(Q, U) \\
& =\sum_{j=1}^{t}\left(\begin{array}{c}
t-1 \\
j-1
\end{array}\right) \ell_{R}\left(\mathrm{H}_{\mathfrak{m}}^{j}(N)\right)+n^{t} e_{0}\left(\left(x_{1}, \ldots, x_{t}\right), U\right) \\
& \geq n^{t} .
\end{aligned}
$$

Thus $\Lambda_{r-t}(M)$ is not finite which implies that $r-t \geq k+1$. Thus $t \leq r-(k+1)$. Consequently, $\mathrm{H}_{\mathfrak{m}}^{i}(U)=0$ for all $i \geq r-k$. Hence $\mathrm{H}_{\mathfrak{m}}^{i}(M) \simeq \mathrm{H}_{\mathfrak{m}}^{i}(N)$ has finite length for all $r-k \leq i \leq r-1$.

Next, we improve a result of Goto and Ozeki [GO11, Theorem 1.1] and generalize it for modules. In order to prove this we recall the following result from [CLT15].

Theorem 4.6. [CLT15, Corollary 4] Let $M$ be a generalized Cohen-Macaulay module. Then, there exists a constant $C$ such that $\operatorname{reg}\left(G_{Q}(M)\right) \leq C$ for all parameter ideals $Q$ for $M$.

Theorem 4.7. Let $(R, \mathfrak{m})$ be a Noetherian local ring and $M$ a finitely generated $R$-module of dimension $r \geq 2$. Then the following conditions are equivalent:

(1) $M$ is a generalized Cohen-Macaulay module;

(2) The set $\Lambda_{i}(M)$ is finite for all $1 \leq i \leq r$;

(3) The set $\Lambda_{i}(M)$ is finite for all $1 \leq i \leq r-\operatorname{depth} M$.

Proof. (1) $\Rightarrow$ (2): Follows from Theorems 4.4 and 4.6.

(2) $\Rightarrow$ (3): This is clear.

(3) $\Rightarrow$ (1): Follows from Lemma 4.5.

We now discuss an example from [GO11] which illustrates the significance of the finiteness of $\Lambda_{i}(M)$ for $i=\operatorname{dim} M-\operatorname{depth} M$.

Example 4.8. [GO11, Example 3.5] Let $(R, \mathfrak{n})$ be a regular local ring of dimension $d \geq 2$ and $X_{1}, \ldots, X_{d}$ a regular system of parameters of $R$. We put $\mathfrak{p}=\left(X_{1}, \ldots, X_{d-1}\right)$ and $D=R / \mathfrak{p}$. 
Let $A=R \ltimes D$ be the idealization of $D$ over $R$. Then $A$ is a Noetherian local ring with the maximal ideal $\mathfrak{m}=\mathfrak{n} \times D, \operatorname{dim} A=d$ and depth $A=1$. By [GO11, Example 3.5]

$$
\Lambda_{i}(A)= \begin{cases}\{n \mid 0<n \in \mathbb{Z}\} & \text { if } i=0 \\ \{0\} & \text { if } 1 \leq i \leq d \text { and } i \neq d-1 \\ \left\{(-1)^{d-1} n \mid 0<n \in \mathbb{Z}\right\} & \text { if } i=d-1\end{cases}
$$

and $\mathrm{H}_{\mathfrak{m}}^{1}(A)\left(\cong \mathrm{H}_{\mathfrak{n}}^{1}(D)\right)$ is not a finitely generated $A$-module. Hence $A$ is not generalized Cohen-Macaulay.

As a consequence of Theorem 4.7 we obtain a characterization of generalized Cohen-Macaulay rings in terms of the coefficients $g_{i}^{K}(Q)$.

Theorem 4.9. Let $(R, \mathfrak{m})$ be a Noetherian local ring of dimension $d \geq 2$ and $K$ an $\mathfrak{m}$-primary ideal of $R$. Then the following conditions are equivalent:

(1) $R$ is generalized Cohen-Macaulay;

(2) $\Lambda_{i}^{K}(R)$ is finite for all $1 \leq i \leq d$;

(3) $\Lambda_{i}^{K}(R)$ is finite for all $1 \leq i \leq d-1$;

(4) $\delta_{i}^{K}(R)$ is finite for all $1 \leq i \leq d-1$.

Proof. By Lemma 2.3(1), (1) is equivalent to the generalized Cohen-Macaulayness of $K$. From (2.1), $\left|\Lambda_{i}(K)\right|=\left|\Lambda_{i}^{K}(R)\right|$ for all $1 \leq i \leq d$. Hence (1) $\Rightarrow$ (2) follows from Theorem 4.7. The implication $(2) \Rightarrow(3) \Rightarrow(4)$ is clear. We show $(4) \Rightarrow(1)$. Since $\delta_{i}^{K}(R)$ is finite, by (2.1),

$$
\left\{e_{i}(Q, K): Q \text { is a parameter ideal of } R \text { and } Q \subseteq K\right\}
$$

is finite for all $1 \leq i \leq d-1$. Therefore by Lemma $4.5, K$ is generalized Cohen-Macaulay. Thus $R$ is generalized Cohen-Macaulay by Lemma 2.3(1).

In the following theorem we give a characterization for $M / \mathrm{H}_{\mathfrak{m}}^{0}(M)$ to be Buchsbaum in terms of $\Lambda_{i}(M)$. See also [GGH ${ }^{+} 15$, Theorem 5.4].

Theorem 4.10. Let $(R, \mathfrak{m})$ be a Noetherian local ring and $M$ a finitely generated $R$-module of dimension $r \geq 2$. Then the following statements are equivalent:

(1) $M / \mathrm{H}_{\mathfrak{m}}^{0}(M)$ is a Buchsbaum R-module;

(2) $\left|\Lambda_{i}(M)\right|=1$ for all $1 \leq i \leq r$;

(3) $\left|\Lambda_{i}(M)\right|=1$ for all $1 \leq i \leq r-\operatorname{depth} M$.

Proof. (1) $\Rightarrow(2)$ : Let $M^{\prime}:=M / \mathrm{H}_{\mathfrak{m}}^{0}(M)$. Since $M^{\prime}$ is Buchsbaum, every parameter ideal $Q$ for $M^{\prime}$ is standard. Hence by [Tru86, Corollary 4.2], $e_{i}\left(Q, M^{\prime}\right)=(-1)^{i} \sum_{j=0}^{r-i}\left(\begin{array}{c}r-i-1 \\ j-1\end{array}\right) \ell_{R}\left(\mathrm{H}_{\mathfrak{m}}^{j}\left(M^{\prime}\right)\right)$ for all $1 \leq i \leq r$. Thus $\left|\Lambda_{i}\left(M^{\prime}\right)\right|=1$ for all $1 \leq i \leq r$. Hence, using (4.5), $\left|\Lambda_{i}(M)\right|=\left|\Lambda_{i}\left(M^{\prime}\right)\right|=1$ for all $1 \leq i \leq r$.

$(2) \Rightarrow(3)$ : This is clear.

(3) $\Rightarrow(1)$ : Let $M^{\prime}:=M / \mathrm{H}_{\mathrm{m}}^{0}(M)$. Since $\left|\Lambda_{i}(M)\right|=\left|\Lambda_{i}\left(M^{\prime}\right)\right|$ by $(4.5),\left|\Lambda_{i}\left(M^{\prime}\right)\right|=1$ for all $1 \leq i \leq r-\operatorname{depth} M$. Hence, by Theorem 4.7, $M^{\prime}$ is a generalized Cohen-Macaulay module $R$-module. This implies that $\widehat{M}^{\prime}$ is a generalized Cohen-Macaulay $\widehat{R}$-module. Since $\operatorname{depth}_{\widehat{R}} \widehat{M^{\prime}}>0$, using [Tru86, Lemma 1.2], we conclude that $M^{\prime}$ is an unmixed module. Hence, by $\left[\mathrm{GGH}^{+} 15\right.$, Theorem 5.4], $M^{\prime}$ is a Buchsbaum $R$-module.

As a consequence we give a sufficient condition for $R / \mathrm{H}_{\mathfrak{m}}^{0}(R)$ to be Buchsbaum in terms of $\Lambda_{i}^{K}(R)$. 
Theorem 4.11. Let $(R, \mathfrak{m})$ be a Noetherian local ring of dimension $d \geq 2$ and $K$ an $\mathfrak{m}$-primary ideal of $R$.

(1) Suppose $\left|\Lambda_{i}^{K}(R)\right|=1$ for all $1 \leq i \leq d-1$. Then $R / H_{\mathfrak{m}}^{0}(R)$ is Buchsbaum.

(2) If $R / \mathrm{H}_{\mathfrak{m}}^{0}(R)$ is Buchsbaum then $\left|\Lambda_{i}^{\mathfrak{m}}(R)\right|=1$ for all $1 \leq i \leq d$.

Proof. (1): From (2.1), $\left|\Lambda_{i}^{K}(R)\right|=\left|\Lambda_{i}(K)\right|$. Hence taking $M=K$ in Theorem 4.10, we get that $K / \mathrm{H}_{\mathfrak{m}}^{0}(K)$ is Buchsbaum. Thus, by Lemma 2.3(2), $R / \mathrm{H}_{\mathfrak{m}}^{0}(R)$ is Buchsbaum.

(2): By Lemma 2.3(3), $\mathfrak{m} / H_{\mathfrak{m}}^{0}(\mathfrak{m})$ is a Buchsbaum $R$-module. Since $\left|\Lambda_{i}^{\mathfrak{m}}(R)\right|=\left|\Lambda_{i}(\mathfrak{m})\right|$, by Theorem 4.10, the result follows.

\section{THE SET $\Delta^{K}(R)$}

For an $R$-module $M$, we set

$$
\Delta_{R}(M)=\left\{e_{1}(I, M) \mid I \text { is an } \mathfrak{m} \text {-primary ideal of } R\right\} .
$$

In [KT15] authors gave a necessary and sufficient condition for the finiteness of the set $\Delta_{R}(R)$. In this section we give an equivalent criterion for the finiteness of the set $\Delta^{K}(R)$ (Theorem 5.7). For this purpose we first give a characterization for the set $\Delta_{R}(M)$ to be finite (Theorem 5.6). We use a bound given by T. Puthenpurakal, [Put03, Theorem 18], to give a sufficient condition for the finiteness of $\Delta_{R}(M)$. In order to obtain a necessary condition we use "induction".

We need few lemmas in order to prove Theorem 5.6. In the following lemma we show that if $\Delta_{R}(M)$ is finite then $\operatorname{dim} M=1$. Proof given here is similar to the proof of [KT15, Lemma 3.1].

Lemma 5.1. Let $(R, \mathfrak{m})$ be a Noetherian local ring and $M$ a finitely generated $R$-module of dimension $r>0$. Suppose $\Delta_{R}(M)$ is a finite set. Then $r=1$.

Proof. Let $I$ be an $\mathfrak{m}$-primary ideal of $R$ and $k \geq 1$ an integer. We have

$$
\ell_{R}\left(M /\left(I^{k}\right)^{n+1} M\right)=e_{0}\left(I^{k}, M\right)\left(\begin{array}{c}
n+r \\
r
\end{array}\right)-e_{1}\left(I^{k}, M\right)\left(\begin{array}{c}
n+r-1 \\
r-1
\end{array}\right)+\ldots+(-1)^{r} e_{r}\left(I^{k}, M\right)
$$

Also,

$$
\ell_{R}\left(M / I^{k n+k} M\right)=e_{0}(I, M)\left(\begin{array}{c}
(k n+k-1)+r \\
r
\end{array}\right)-e_{1}(I, M)\left(\begin{array}{c}
(k n+k-1)+r-1 \\
r-1
\end{array}\right)
$$

Note that

$\left(\begin{array}{c}k n+k+r-1 \\ r\end{array}\right)=k^{r}\left(\begin{array}{c}n+r \\ r\end{array}\right)+\left(k^{r-1}-k^{r}\right)\left(\frac{r-1}{2}\right)\left(\begin{array}{c}n+r-1 \\ r-1\end{array}\right)+$ lower degree terms and $\left(\begin{array}{c}k n+k+r-2 \\ r-1\end{array}\right)=k^{r-1}\left(\begin{array}{c}n+r-1 \\ r-1\end{array}\right)+$ lower degree terms.

Comparing (5.1) and (5.2), we get

$$
\begin{aligned}
e_{0}\left(I^{k}, M\right) & =k^{r} e_{0}(I, M) \text { and } \\
e_{1}\left(I^{k}, M\right) & =\frac{r-1}{2} e_{0}(I, M) k^{r}+\frac{2 e_{1}(I, M)-(r-1) e_{0}(I, M)}{2} k^{r-1} .
\end{aligned}
$$

Since $\Delta_{R}(M)$ is a finite set, the set $\left\{e_{1}\left(I^{k}, M\right) \mid k \geq 1\right.$ is an integer $\}$ is also finite. Hence using (5.3), we get $r=1$.

In view of Lemma 5.1, we assume that $r=1$ while examining the finiteness of the set $\Delta_{R}(M)$. Now we recall the following theorem from [KT15] which will be used in this section. 
Theorem 5.2. [KT15, Theorem 1.1] Let $(R, \mathfrak{m})$ be a Noetherian local ring of dimension $d>0$. Then the following conditions are equivalent:

(1) $\Delta_{R}(R)$ is a finite set;

(2) $d=1$ and $R / \mathrm{H}_{\mathfrak{m}}^{0}(R)$ is analytically unramified.

To discuss the finiteness of $\Delta_{R}(M)$, we first provide bounds on this set in the following proposition.

Proposition 5.3. Let $(R, \mathfrak{m})$ be a Noetherian local ring of dimension one and $M$ a finitely generated $R$-module of dimension one. Then

(1) $\inf \Delta_{R}(M)=-\ell_{R}\left(\mathrm{H}_{\mathfrak{m}}^{0}(M)\right)$.

(2) $\sup \Delta_{R}(M) \leq \ell_{R^{\prime}}\left(\overline{R^{\prime}} / R^{\prime}\right) \mu_{R^{\prime}}\left(M^{\prime}\right)$, where $R^{\prime}:=R / \mathrm{H}_{\mathfrak{m}}^{0}(R)$ and $M^{\prime}:=M / \mathrm{H}_{\mathfrak{m}}^{0}(M)$. Here $\overline{R^{\prime}}$ denotes the integral closure of $R^{\prime}$ in its total ring of fractions.

Proof. (1): Let $c=\inf \Delta_{R}(M)$. By (4.5), for every m-primary ideal $I$ in $R$,

$$
e_{1}(I, M)=e_{1}\left(I, M^{\prime}\right)-\ell_{R}\left(\mathrm{H}_{\mathfrak{m}}^{0}(M)\right) \text {. }
$$

Since $M^{\prime}$ is Cohen-Macaulay, $e_{1}\left(I, M^{\prime}\right) \geq 0$ by Northcott's inequality for modules (see [Fil67, p. 218]). Thus $e_{1}(I, M) \geq-\ell_{R}\left(\mathrm{H}_{\mathrm{m}}^{0}(M)\right)$ for every m-primary ideal $I$ in $R$ which implies that $c \geq-\ell_{R}\left(\mathrm{H}_{\mathfrak{m}}^{0}(M)\right)$. Let $Q=(x)$ be a parameter ideal for $M$. Then, by $(5.4), e_{1}(Q, M)=$ $-\ell_{R}\left(\mathrm{H}_{\mathfrak{m}}^{0}(M)\right)$. Hence $c=-\ell_{R}\left(\mathrm{H}_{\mathfrak{m}}^{0}(M)\right)$.

(2): Let $C=\sup \Delta_{R}(M)$. Note that $M^{\prime}$ is a maximal Cohen-Macaulay $R^{\prime}$-module. Hence, for every m-primary ideal $I$ of $R$, we have

$$
\begin{array}{rlrl}
e_{1}(I, M) & \leq e_{1}\left(I, M^{\prime}\right), & & (\text { by }(5.4)) \\
& =e_{1}\left(I R^{\prime}, M^{\prime}\right) & \\
& \leq e_{1}\left(I R^{\prime}, M^{\prime}\right)+e_{1}\left(I R^{\prime}, \operatorname{Syz}_{1}^{R^{\prime}}\left(M^{\prime}\right)\right) & & \left(\text { as } \operatorname{Syz}_{1}^{R^{\prime}}\left(M^{\prime}\right) \text { is a Cohen-Macaulay } R^{\prime} \text {-module }\right) \\
& \leq e_{1}\left(I R^{\prime}, R^{\prime}\right) \mu_{R^{\prime}}\left(M^{\prime}\right) & & (\text { by }[\text { Put03, Proposition 17] }) \\
& \leq \ell_{R^{\prime}}\left(\overline{R^{\prime}} / R^{\prime}\right) \mu_{R^{\prime}}\left(M^{\prime}\right) & & (\text { by [KT15, Theorem 1.2]). }
\end{array}
$$

Hence $C \leq \ell_{R^{\prime}}\left(\overline{R^{\prime}} / R^{\prime}\right) \mu_{R^{\prime}}\left(M^{\prime}\right)$.

In order to obtain an upper bound on the set $\Delta_{R}(M)$, the ring $R$ having dimension one in Proposition 5.3 is not a restrictive condition as we may pass to $R / \operatorname{Ann}_{R}(M)$, if needed, and assume that $\operatorname{dim} R=1$.

Proposition 5.4. Let $(R, \mathfrak{m})$ be a Noetherian local ring and $M$ a Cohen-Macaulay $R$-module of dimension one. For nonzero modules $N$ and $C$, consider the exact sequence

$$
0 \longrightarrow N \longrightarrow M \longrightarrow C \longrightarrow 0 \text {. }
$$

For an m-primary ideal $I$ in $R$, the following statements hold true.

(1) If $\operatorname{dim} C=0$, then $e_{1}(I, M) \geq e_{1}(I, N)-\ell_{R}(C)$.

(2) If $\operatorname{dim} C=1$, then $e_{1}(I, M) \geq e_{1}(I, N)+e_{1}(I, C) \geq e_{1}(I, N)-\ell_{R}\left(\mathrm{H}_{\mathfrak{m}}^{0}(C)\right)$.

Proof. Tensoring (5.5) with $R / I^{n+1}$, we get an exact sequence

$$
0 \longrightarrow K_{I, n+1} \longrightarrow \frac{N}{I^{n+1} N} \longrightarrow \frac{M}{I^{n+1} M} \longrightarrow \frac{C}{I^{n+1} C} \longrightarrow 0,
$$

where $K_{I, n+1}$ (depends on $I$ and $n$ ) is some $R$-module of finite length. Therefore

$$
\ell_{R}\left(K_{I, n+1}\right)-\ell_{R}\left(\frac{N}{I^{n+1} N}\right)+\ell_{R}\left(\frac{M}{I^{n+1} M}\right)-\ell_{R}\left(\frac{C}{I^{n+1} C}\right)=0 .
$$


This implies that $\ell_{R}\left(K_{I, n+1}\right)$ is a polynomial, for $n \gg 0$, of degree at most one. Let $\ell_{R}\left(K_{I, n+1}\right)=$ $a_{I}(n+1)+b_{I}$, for $n \gg 0$, where $a_{I}$ and $b_{I}$ are some integers. Since $M$ is Cohen-Macaulay, $N$ is a Cohen-Macaulay module of dimension one. Hence, by using [BH93, Corollary 4.7.7], we get $a_{I}=0$ and hence $\ell_{R}\left(K_{I, n+1}\right)=b_{I}$ for $n \gg 0$.

5.4(1): Suppose that $\operatorname{dim} C=0$. Then $I^{n} C=0$ for $n \gg 0$. Hence from (5.6), we get that

$$
e_{1}(I, M)=b_{I}+e_{1}(I, N)-\ell_{R}(C) \geq e_{1}(I, N)-\ell_{R}(C) .
$$

5.4(2): Again using (5.6), we get

$$
\begin{aligned}
e_{1}(I, M) & =b_{I}+e_{1}(I, N)+e_{1}(I, C) \\
& \geq e_{1}(I, N)+e_{1}(I, C) \\
& \geq e_{1}(I, N)-\ell_{R}\left(\mathrm{H}_{\mathfrak{m}}^{0}(C)\right)
\end{aligned}
$$

In the following lemma we give a necessary condition for the finiteness of the set $\Delta_{R}(M)$ if $M$ is a cyclic module of dimension one.

Lemma 5.5. Let $(R, \mathfrak{m})$ be a Noetherian local ring and $M=R x$ a Cohen-Macaulay $R$-module of dimension one. Suppose $\Delta_{R}(M)$ is finite. Then $R / \operatorname{Ann}_{R}(M)$ is analytically unramified.

Proof. Note that $M \cong R / \operatorname{Ann}_{R}(x)$. Let $B:=R / \operatorname{Ann}_{R}(x)$. Since $\ell_{R}\left(B / I^{n} B\right)=\ell_{B}\left(B / I^{n} B\right)$ for any $\mathfrak{m}$-primary ideal $I$ in $R, e_{1}(I, B)=e_{1}(I B, B)$. Since every $\mathfrak{m} B$-primary ideal in $B$ is of the form $I B$ for some m-primary ideal $I$ in $R$, finiteness of the set $\Delta_{R}(M)$ implies that the set $\Delta_{B}(B)$ is finite. Therefore, by Theorem $5.2, B$ is analytically unramified.

Now we give an equivalent criterion for the finiteness of the set $\Delta_{R}(M)$.

Theorem 5.6. Let $(R, \mathfrak{m})$ be a Noetherian local ring and $M$ a finitely generated $R$-module of dimension $r>0$. Let $R^{\prime}=R / \mathrm{H}_{\mathfrak{m}}^{0}(R)$ and $M^{\prime}=M / \mathrm{H}_{\mathfrak{m}}^{0}(M)$. Then the following conditions are equivalent:

(1) $\Delta_{R}(M)$ is a finite set;

(2) $r=1$ and $R^{\prime} / \operatorname{Ann}_{R^{\prime}}\left(M^{\prime}\right)$ is analytically unramified.

Proof. (1) $\Rightarrow$ (2): Since $\Delta_{R}(M)$ is finite, by Lemma 5.1, $r=1$. Thus $M^{\prime}$ is a Cohen-Macaulay $R^{\prime}$-module of dimension one. From (5.4) it follows that $\left|\Delta_{R}(M)\right|=\left|\Delta_{R}\left(M^{\prime}\right)\right|$. This implies that $\Delta_{R}\left(M^{\prime}\right)$ is a finite set. Since $e_{1}\left(I, M^{\prime}\right)=e_{1}\left(I R^{\prime}, M^{\prime}\right)$, we get that $\Delta_{R^{\prime}}\left(M^{\prime}\right)$ is a finite set. Let $M^{\prime}=R^{\prime} x_{1}+R^{\prime} x_{2}+\cdots+R^{\prime} x_{m}$, where $0 \neq R^{\prime} x_{i} \subseteq M^{\prime}$ is a $R^{\prime}$-submodule of $M^{\prime}$. Set $N_{i}:=R^{\prime} x_{i}$. Since $M^{\prime}$ is Cohen-Macaulay, $N_{i}$ is a Cohen-Macaulay $R^{\prime}$-module of dimension one. Hence for every m-primary ideal $I$ in $R, e_{1}\left(I R^{\prime}, N_{i}\right) \geq 0$ and by Proposition 5.4,

$$
e_{1}\left(I R^{\prime}, N_{i}\right) \leq e_{1}\left(I R^{\prime}, M^{\prime}\right)+c_{i},
$$

for some nonnegative integer $c_{i}$ which is independent of $I$. Thus finiteness of the set $\Delta_{R^{\prime}}\left(M^{\prime}\right)$ implies that the set $\Delta_{R^{\prime}}\left(N_{i}\right)$ is finite for every $i$. Hence, by Lemma 5.5, $R^{\prime} / \operatorname{Ann}_{R^{\prime}}\left(R^{\prime} x_{i}\right)$ is analytically unramified for each $i$. Let $I_{i}=\operatorname{Ann}_{R^{\prime}}\left(R^{\prime} x_{i}\right)$. Since $\widehat{R}^{\prime} / I_{i} \widehat{R}^{\prime}$ is reduced for each $i$, $\widehat{R}^{\prime} /\left(\bigcap_{i=1}^{m} I_{i} \widehat{R}^{\prime}\right)$ is reduced. Also, as $\widehat{R}^{\prime}$ is a flat $R^{\prime}$-module,

$$
\left.\widehat{\operatorname{Ann}_{R^{\prime}}\left(M^{\prime}\right.}\right)=\operatorname{Ann}_{R^{\prime}}\left(M^{\prime}\right) \widehat{R}^{\prime}=\left(\bigcap_{i=1}^{m} I_{i}\right) \widehat{R^{\prime}}=\bigcap_{i=1}^{m} I_{i} \widehat{R^{\prime}}
$$

Hence $\widehat{R^{\prime}} / \widehat{\operatorname{Ann} n_{R^{\prime}} M^{\prime}} \cong\left(\widehat{\frac{R^{\prime}}{\operatorname{Ann}_{R^{\prime}} M^{\prime}}}\right)$ is reduced. Thus $R^{\prime} / \operatorname{Ann}_{R^{\prime}}\left(M^{\prime}\right)$ is analytically unramified. 
$(2) \Rightarrow(1):$ Since $\operatorname{dim} R^{\prime} / \operatorname{Ann}_{R^{\prime}}\left(M^{\prime}\right)=\operatorname{dim} M^{\prime}=1$ and $R^{\prime} / \operatorname{Ann}_{R^{\prime}}\left(M^{\prime}\right)$ is analytically unramified, by Proposition 5.3, $\frac{R^{\prime}}{\mathrm{Ann}_{R^{\prime}}\left(M^{\prime}\right)}\left(M^{\prime}\right)$ is finite. This implies that $\Delta_{R^{\prime}}\left(M^{\prime}\right)$ is finite. Hence by (5.4), $\Delta_{R}(M)$ is a finite set.

As a consequence we give an equivalent criterion for the finiteness of the set $\Delta^{K}(R)$.

Theorem 5.7. Let $(R, \mathfrak{m})$ be a Noetherian local ring of dimension $d>0$ and $K$ an $\mathfrak{m}$-primary ideal of $R$. Then the following conditions are equivalent:

(1) $\Delta^{K}(R)$ is a finite set;

(2) $d=1$ and $R / H_{\mathfrak{m}}^{0}(R)$ is analytically unramified.

Proof. From (2.1), $\left|\Delta^{K}(R)\right|=\left|\Delta_{R}(K)\right|$. Let $R^{\prime}=R / H_{\mathfrak{m}}^{0}(R)$. Since $\operatorname{Ann}_{R^{\prime}}\left(K R^{\prime}\right)=0$, using Theorem 5.6 we get the result.

Remark 5.8. Suppose $d=1$ and $R / H_{\mathfrak{m}}^{0}(R)$ is analytically unramified. Then by Theorems 5.2 and 5.7, the sets $\Delta_{R}(R)$ and $\Delta^{K}(R)$, respectively, are finite. Hence, from (2.3), the set $\left\{f_{0}^{K}(I) \mid I\right.$ is an $\mathfrak{m}$-primary ideal of $\left.R\right\}$ is finite.

In [KT15, Corollary 2.4], authors gave a description of the set $\Delta_{R}(R)$. In what follows we will give a description of the set $\Delta^{K}(R)$. Recall that a reduction of an ideal $I$ is an ideal $J \subseteq I$ such that $I^{n+1}=J I^{n}$ for some $n \geq 0$. A minimal reduction of $I$ is a reduction of $I$ which is minimal with respect to inclusion. For a minimal reduction $J$ of $I$, reduction number of $I$ with respect to $J$, denoted by $r_{J}(I)$, is the least non-negative integer $n$ such that $I^{n+1}=J I^{n}$.

Theorem 5.9. Let $(R, \mathfrak{m})$ be a Cohen-Macaulay local ring of dimension one with infinite residue field.

(1) For a maximal Cohen-Macaulay module $M$

$$
\Delta_{R}(M) \subseteq\left\{\ell_{R}(N / M): M \subseteq N \subseteq S^{-1} M, N \text { is a finitely generated R-module }\right\},
$$
where $S=\{x \in R: x$ is $R$-regular $\}$.

(2) For an $\mathfrak{m}$-primary ideal $K$ in $R$,

$$
\Delta^{K}(R)=\left\{\ell_{R}(K B / K)-\ell_{R}(R / K): R \subseteq B \subseteq \bar{R}, B \text { is a finitely generated } R \text {-module }\right\} .
$$

Further, $\sup \Delta^{K}(R)=\ell_{R}(K \bar{R} / K)-\ell_{R}(R / K)$.

Proof. (1): Let $I$ be an m-primary ideal in $R$. Let $J=(x) \subseteq I$ be a minimal reduction of $I$. Since $R$ (resp. $M$ ) is Cohen-Macaulay, $x$ is $R$-(resp. $M$-)regular. We set

$$
\frac{I^{n}}{x^{n}}=\left\{\frac{a}{x^{n}}: a \in I^{n}\right\} \subseteq S^{-1} R .
$$

Let $s=r_{J}(I)$ and $N=M\left[\frac{I}{x}\right] \subseteq S^{-1} M$. Then $M \subseteq N=\bigcup_{n \geq 0} \frac{I^{n} M}{x^{n}}=\frac{I^{n} M}{x^{n}} \cong I^{n} M$ for $n \geq s$. Thus $N$ is a finitely generated $R$-module. We claim that $e_{1}(I, M)=\ell_{R}(N / M)$. We have

$$
\begin{aligned}
\ell_{R}\left(\frac{M}{I^{n+1} M}\right) & =\ell_{R}\left(\frac{M}{J^{n+1} M}\right)-\ell_{R}\left(\frac{I^{n+1} M}{J^{n+1} M}\right) & \\
& =e_{0}(I, M)(n+1)-\ell_{R}\left(\frac{I^{n+1} M}{J^{n+1} M}\right) & \text { for } n \gg 0 .
\end{aligned}
$$

This implies that $e_{1}(I, M)=\ell_{R}\left(\frac{I^{n+1} M}{J^{n+1} M}\right)$ for $n \gg 0$. Since $\frac{I^{n+1} M}{J^{n+1} M} \cong \frac{N}{M}$ for $n \gg 0, e_{1}(I, M)=$ $\ell_{R}(N / M)$.

(2): Let $\Gamma(R):=\left\{\ell_{R}(K B / K): R \subseteq B \subseteq \bar{R}, B\right.$ is a finitely generated $R$-module $\}$. First we show that $\Delta_{R}(K)=\Gamma(R)$. By part $(1), e_{1}(I, K)=\ell_{R}(N / K)$, where $N=K\left[\frac{I}{x}\right]$ and $(x)$ is a 
minimal reduction of $I$. Put $B=R\left[\frac{I}{x}\right]$. Let $s=r_{(x)}(I)$. Then $B=\frac{I^{n}}{x^{n}} \cong I^{s}$ for all $n \geq s$. Thus $B$ is a finitely generated $R$-module which implies that $B \subseteq \bar{R}$. Also, $K B=K\left[\frac{I}{x}\right]=N$. Hence $e_{1}(I, K)=\ell_{R}(K B / K) \in \Gamma(R)$.

Now, let $R \subseteq B \subseteq \bar{R}$ and $B$ is finitely generated $R$-module. Then there exists a nonzerodivisor $x \in R$ such $x B \subseteq R$. Let $I=x B$. Then $I$ is an m-primary ideal in $R$ and $I^{2}=x I$. Hence $R\left[\frac{I}{x}\right]=\frac{I}{x}=B$. A similar argument as above shows that $e_{1}(I, K)=\ell_{R}(K B / K)$. Hence $\Gamma(R) \subseteq \Delta_{R}(K)$. Thus $\Gamma(R)=\Delta_{R}(K)$. Therefore using (2.1), (5.7) follows.

Let $C:=\sup \Delta^{K}(R)$. From (5.7) it follows that $C \leq \ell_{R}(K \bar{R} / K)-\ell_{R}(R / K)$. Hence in order to prove the second assertion we may assume that $C$ is finite. Then, by Theorem 5.7, $R$ is analytically unramified and hence $\bar{R}$ is a finite $R$-module. Again using (5.7), we get $C \geq$ $\ell_{R}(K \bar{R} / K)-\ell_{R}(R / K)$.

Remark 5.10. (1) The containment in Theorem 5.9(1) can be strict. Let $R$ be a CohenMacaulay local ring of dimension one and $I$ an $\mathfrak{m}$-primary ideal. Choose an integer $t$ such that $e_{1}(I, R)$ is not divisible by $t$. Let $M=R^{t}$. Then $e_{1}(J, M)=t e_{1}(J, R)$ for every m-primary ideal $J$ in $R$. By [KT15, Corollary 2.4], $e_{1}(I, R)=\ell_{R}(B / R)$, for a finite $R$-module $B$ such that $R \subseteq B \subseteq S^{-1} R$. Now, $R^{t} \subseteq N:=B \oplus R \oplus \cdots \oplus R \subseteq\left(S^{-1} R\right)^{t}$ and $N$ is a finite $R$-module. Also, $\ell_{R}(N / M)=\ell_{R}(B / R)=e_{1}(I, R)$. Suppose there exists an m-primary ideal $J$ in $R$ such that $e_{1}(J, M)=\ell_{R}(N / M)=e_{1}(I, R)$. Then $t e_{1}(J, R)=e_{1}(I, R)$ which is a contradiction. This implies that the containment in Theorem 5.9(1) can be strict.

(2) Let $R$ be a Cohen-Macaulay local ring of dimension one and $M=R^{t}$. In this case, $\Delta_{R}(M)=\left\{t e_{1}(I, R): I\right.$ is an m-primary ideal in $\left.R\right\}$. Hence, by [KT15, Theorem 1.2], $\sup \Delta_{R}(M)=t \ell_{R}(\bar{R} / R)=\ell_{R}(\bar{R} / R) \mu_{R}(M)$, which shows that the bound in Proposition 5.3(2) can be achieved.

\section{ACKNOWLEDGEMENTS}

The first author is grateful to Prof. Santiago Zarzuela for insightful discussions on section 5. His ideas were helpful to get the results in section 5. She thanks the Institute of Mathematical Sciences (IMSc), Chennai for supporting her travel to the Centre de Recerca Matemàtica (CRM), Barcelona during which some part of the work is done. She also thanks CRM for providing local hospitality during the visit. The second author is indebted to her advisor Prof. Anupam Saikia for the encouragement to pursue this work. She also thanks the Indian Institute of Technology, Guwahati for granting the Ph.D. scholarship and IMSc for its hospitality where a significant part of this work was discussed.

\section{REFERENCES}

[AB58] M. Auslander and D. Buchsbaum, Codimension and multiplicity, Ann. of Math. 68 (1958), 625-657.

[BH93] W. Bruns and J. Herzog, Cohen-Macaulay rings, revised edition, Cambridge University Press, Cambridge, 1998.

[D'cr13] C. D'Cruz, On the homology and fiber cone of ideals, Comm. Algebra 41 (2013), 42274247.

[CLT15] N. T. Cuong, N. T. Long and H. L. Truong, Uniform bounds in sequentially generalized Cohen-Macaulay modules, Vietnam J. Math. 43 (2015), 343-356.

[CST78] N. T. Cuong, P. Schenzel and N. V. Trung, Verallgemeinerte Cohen-Macaulay-Moduln, Math. Nachr. 85 (1978), 57-73.

[Fil67] J. P. Fillmore, On the coefficients of the Hilbert-Samuel polynomial, Math. Z. 97 (1967), 212-228. 
[GGH ${ }^{+}$10] L. Ghezzi, S. Goto, J. Hong, K. Ozeki, T. T. Phuong and W. V. Vasconcelos, CohenMacaulayness versus the vanishing of the first Hilbert coefficient of parameter ideals, J. London Math. Soc. 81 (2010), 679-695.

$\left[\mathrm{GGH}^{+}\right.$15] L. Ghezzi, S. Goto, J. Hong, K. Ozeki, T. T. Phuong and W. V. Vasconcelos, The Chern numbers and Euler characteristics of modules, Acta Math. Vietnamica 40 (2015), 37-60.

[GZT07] Y. Gu, G. Zhu and Z. Tang, On Hilbert coefficients of filtrations, Chin. Ann. Math. 28B(5) (2007), 543-554.

[GN03] S. Goto and K. Nishida, Hilbert coefficients and Buchsbaumness of associated graded rings, J. Pure and Applied Algebra 181 (2003), 61-74.

[GO11] S. Goto and K. Ozeki, Uniform Bounds for Hilbert coefficients of parameters, Contemporary Mathematics 555 (2011), 97-118.

[HH11] F. Hayasaka and E. Hyry, On the Buchsbaum-Rim function of a parameter module, J. Algebra 327 (2011), 307-315.

[JV05a] A. V. Jayanthan and J. K. Verma, Hilbert coefficients and depth of fiber cones, J. Pure and Applied Algebra 201 (2005), 97-115.

[JV05b] A. V. Jayanthan and J. K. Verma, Fiber cones of ideals with almost minimal multiplicity, Nagoya Math. J. 177 (2005), 155-179.

[KT15] A. Koura and N. Taniguchi, Bounds for the first Hilbert coefficients of $\mathfrak{m}$-primary ideals, Tokyo J. Math. 38 (2015), 125-133.

[LT06] C. H. Linh and N. V. trung, Uniform bounds in generalized Cohen-Macaulay rings, J. Algebra 304 (2006), 1147-1159.

[MSV11] M. Mandal, B. Singh and J. K. Verma, On some conjectures about the Chern numbers of filtrations, J. Algebra 325 (2011), 147-162.

[Mat06] H. Matsumura, Commutative Ring Theory, second edition, Cambridge University Press, Cambridge, 1989.

[Put03] T. J. Puthenpurakal, Hilbert coefficients of a Cohen-Macaulay module, J. Algebra 264 (2003), 82-97.

[RV10] M. Rossi and G. Valla, Hilbert Functions of Filtered Modules, Lecture Notes of the Unione Matematica Italiana, vol. 9. Berlin, (2010).

[Sal16] K. Saloni, On Hilbert coefficients of parameter ideals and Cohen-Macaulayness, to appear in J. Commutative Algebra.

[Tru86] N. V. Trung, Toward a theory of generalized Cohen-Macaulay modules, Nagoya Math. J. 102 (1986), 1-49.

[ZGT08] G. Zhu, Y. Gu and Z. Tang, Hilbert coefficients of filtrations with almost maximal depth, J. Math. Research and Exposition 28(4) (2008).

Dipartimento Di Matematica, Università Di Genova, Via Dodecaneso 35, 16146, Genova, Italy

E-mail address: masuti@dima.unige.it,masuti.shree@gmail.com

Department of MATHEMATICS, IIT GUWAHATI, GUWAHATI, AsSAM 781039, INDiA

E-mail address: saloni.kumari@iitg.ac.in 